\title{
El acuífero costero La Yarada, después de 100 años de explotación como sustento de una agricultura en zonas áridas: una revisión histórica
}

\author{
The La Yarada coastal aquifer, after 100 years of exploitation \\ as a sustenance for agriculture in arid zones: a historical review
}

\author{
Edwin Pino $V^{1 *}$
}

\section{RESUMEN}

Este artículo es una revisión histórica sobre los sucesos ocurridos en la explotación del acuífero costero La Yarada, emplazado en una zona árida, ubicada en la cabecera del desierto de Atacama. Se ha identificado el período en el que la explotación ha sido en régimen equilibrado (recarga - extracciones) hasta la década de los 70 y no equilibrado a partir de los 80 . Asimismo, se determinan las medidas de gestión favorables y desfavorables en la conservación del sistema acuífero. Se plantean medidas ingenieriles a futuro, conducentes a la recuperación y conservación del acuífero, que consisten en la incorporación de barreras hidráulicas.

Palabras clave: acuífero La Yarada, explotación equilibrada, explotación no equilibrada.

\section{ABSTRACT}

This article is a historical review of the events that occurred in the exploitation of the La Yarada coastal aquifer, located in an arid zone, located at the head of the Atacama Desert. The period in which exploitation has been in a balanced regime (recharge - extractions) until the 70s and unbalanced since the 80s has been identified. It also identifies favorable and unfavorable management measures in the conservation of the aquifer system. Engineering measures were identified in the future, leading to the recovery and conservation of the aquifer, which consist of the incorporation of hydraulic barriers.

Key words: La Yarada aquifer, balanced exploitation, unbalanced exploitation.

\section{Introducción y revisión bibliográfica}

En algunas regiones de nuestro planeta se han examinado los cambios climáticos y ambientales durante los últimos 2 siglos, utilizando registros sistemáticos de precipitaciones e información indirecta sobre lagos y ríos, y la ocurrencia de hambrunas y sequías (Nicholson, 2001). Un estimado de ocho a diez millones de kilómetros cúbicos $\left(\mathrm{km}^{3}\right)$ de agua subterránea fresca representa la mayor parte del total de agua dulce líquida en la Tierra, o para ser más precisos, aproximadamente del 98 al 99\% de ella, según lo mostrado en la Figura 1 (Shiklomanov \& Rodda, 2004). En comparación, el volumen global de agua dulce en los lagos es inferior al $1 \%$ del volumen total de agua dulce subterránea (Shiklomanov \& Rodda, 2004; Schneider et al., 2011; Genda, 2016).

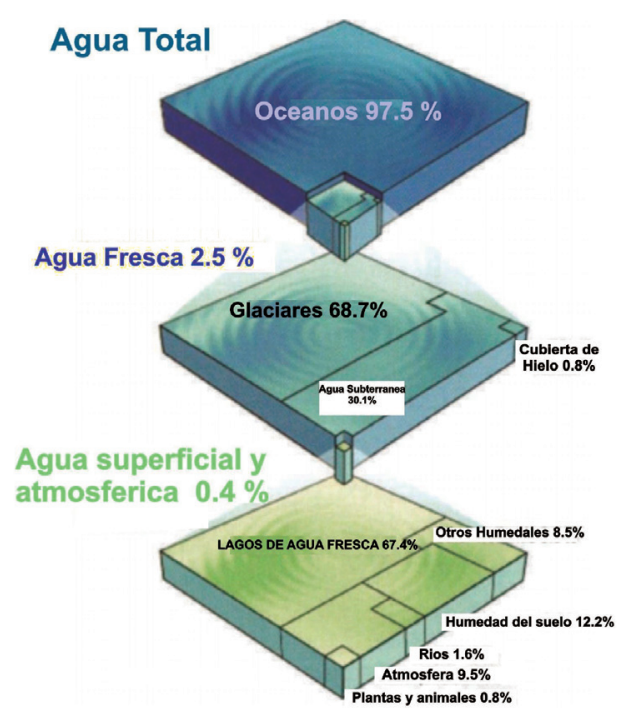

Figura 1. Distribución global del agua en el mundo.

\footnotetext{
$1 \quad$ Universidad Nacional Jorge Basadre Grohmann. Tacna, Perú

* Autor por correspondencia: epino68@hotmail.com, epinov@unjbg.edu.pe
} 
Cuando se trata del cambio climático, el agua subterránea se vuelve particularmente importante. De hecho, cuanto más amenaza el cambio climático el agua superficial a través de las sequías y la salinización, más necesario se hace comprender y administrar mejor los recursos de agua subterránea (Margat \& Gun, 2013). Sin duda, el desafío de lograr una forma de gestión racional y sustentable sobre un acuífero representa, hoy en día, una clara demanda de caminos para la concertación institucional y para la definición de actuaciones (Montaño et al., 1998). Las percepciones relacionadas con el agua subterránea a menudo se basaban en la imaginación, o incluso en la mitología, en lugar de la observación científica (Margat \& Gun, 2013).

La recarga del sistema acuífero sustenta los recursos de agua subterránea de los cuales existe una dependencia mundial para el agua potable y la agricultura bajo riego (Taylor et al., 2012). La dependencia del agua subterránea ha aumentado mucho en América del Sur durante los últimos 20 años, en parte como respuesta a los crecientes costos y otras restricciones para almacenar y tratar las aguas superficiales, y en parte porque ahora las ventajas económicas del uso del agua subterránea se comprenden mejor. Sin embargo, el agua subterránea extraída de los acuíferos es difícil de estimar porque la mayoría proviene de pozos privados y públicos no controlados (Arújo et al., 1999). La vulnerabilidad de las aguas subterráneas al uso excesivo y la degradación de su calidad no se entendió ampliamente sino hasta hace poco tiempo. Debido a la dificultad para estimar las variaciones de almacenamiento del agua subterránea estacional usando observaciones puntuales de los niveles, no se sabe con certeza si los modelos actuales sobreestiman o subestiman las variaciones reales (Döll et al., 2012).

La capacidad existente para la aplicación sobre un acuífero de diferentes tipos de aprovechamientos como el hidrogeológico termal, municipal, industrial o agrícola, lo transforman, por un lado, en un riesgo potencial de sobreexplotación de un recurso natural, y por otro en un factor de riesgo para el desencadenamiento de complejos conflictos sociales (Babaye et al., 2018). En el acuífero costero La Yarada, la crisis de gobernabilidad y gobernanza en el uso del agua subterránea es un elemento que contribuye al agotamiento y deterioro de su calidad, por procesos de intrusión marina (Pino et al., 2018). Aún más, se ha provocado que la calidad del agua pase de aceptable a mala, evidenciando un deterioro que está relacionado con el incremento de los volúmenes de explotación y la disminución de la recarga secundaria de agua de buena calidad (Pino \& Coarita, 2018).

En todo el período de explotación del acuífero, que data de más de 100 años, se tiene información de tres balances hídricos realizados (Tabla 1). Las instituciones responsables de los balances anuales reportados son el Instituto Nacional de Investigación y Fomento Minero (INIFM) en el año 1965 con registro de $36 \mathrm{Hm}^{3}$ en balance positivo, Proyecto Especial Tacna, Instituto Nacional de Desarrollo (PET-INADE) en 1989 con $-15.5 \mathrm{Hm}^{3}$ en balance negativo y el Instituto Geológico Minero y Metalúrgico (INGEMMET) en el año 2009 con $-44.0 \mathrm{Hm}^{3}$ en balance negativo. Posterior a 2009 no se reportan balances en el acuífero.

En la cuenca Caplina, donde se encuentra emplazado este acuífero, se determinaron las fuentes con mayor influencia sobre la calidad del agua. Estas son las geotermales Aruma y Paralocos que emanan aguas con alto contenido de arsénico, plomo y sodio en concentraciones por encima de los valores del Estándar de Calidad Ambiental (ECA), incluso el trasvase de aguas de la fuente Barroso Chico hacia la quebrada Ancoma con alto contenido de hierro (Pino et al., 2017). Este acuífero, al estar ubicado en entornos geológicamente activos, tiene una distribución de tipos de agua que a menudo se ve influida por procesos geológicos a largo plazo (por ejemplo, las fluctuaciones del nivel del mar). Desde el punto de vista de la gestión del agua, los acuíferos costeros son vulnerables a la salinización por intrusión marina (Post, 2005).

En este trabajo buscamos revisar la historia de más de un siglo de explotación del acuífero costero La Yarada como sustento de una agricultura en zonas áridas, además de determinar cuál será el futuro respecto a su explotación y las condiciones de esta en términos de cantidad y calidad.

Tabla 1. Balance hídrico en el acuífero.

\begin{tabular}{lccc}
\hline \multicolumn{1}{c}{ Año } & 1965 & 1989 & 2009 \\
\hline Q explotado $\left(\mathrm{Hm}^{3}\right)$ & 27.0 & 68.0 & 97.0 \\
Q recarga $\left(\mathrm{Hm}^{3}\right)$ & 63.0 & 52.5 & 53.0 \\
Balance $\left(\mathrm{Hm}^{3}\right)$ & 36.0 & 15.5 & -44.0 \\
\hline Fuente & INIFM & PET-INADE & INGEMMET
\end{tabular}




\section{Metodología}

Según las características de este caso, pudimos efectuar una revisión de los documentos existentes en más de un siglo de explotación del acuífero costero La Yarada, ubicado en la región Tacna al sur del Perú (Figura 2). Se consultaron estudios académicos y material oficial de dominio público de instituciones académicas, tecnológicas y científicas. Se busca explicar, mediante una revisión histórica, el funcionamiento del acuífero desde los puntos de vista ingenieriles y de gestión. La información fue analizada y se reportó la discusión y conclusiones sobre esos más de 100 años de explotación. En la Figura 3 se muestra la evolución histórica de la superficie regada y los volúmenes explotados en el acuífero.

\section{Los inicios entre los años 1900 y 1910}

Entre 1900 y 1910 se realizaron las primeras exploraciones, orientadas al petróleo, y fue en 1910 cuando se perforó el primer pozo de explotación de agua subterránea, a cargo de la denominada Junta de Adelanto de Arica (Zagal et al., 2015). No se registraron actividades de intervención en el sistema acuífero posteriores a 1928, fecha de culminación de la ocupación chilena en Tacna.

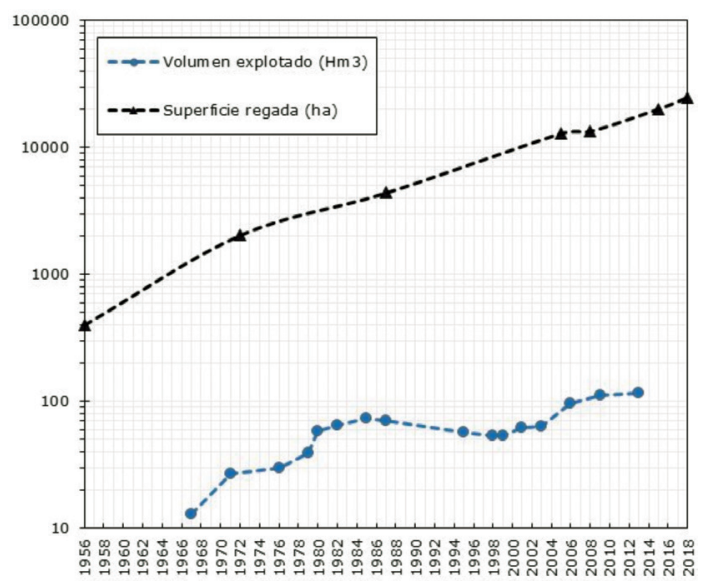

Figura 3. Evolución de la superficie regada y los volúmenes explotados.
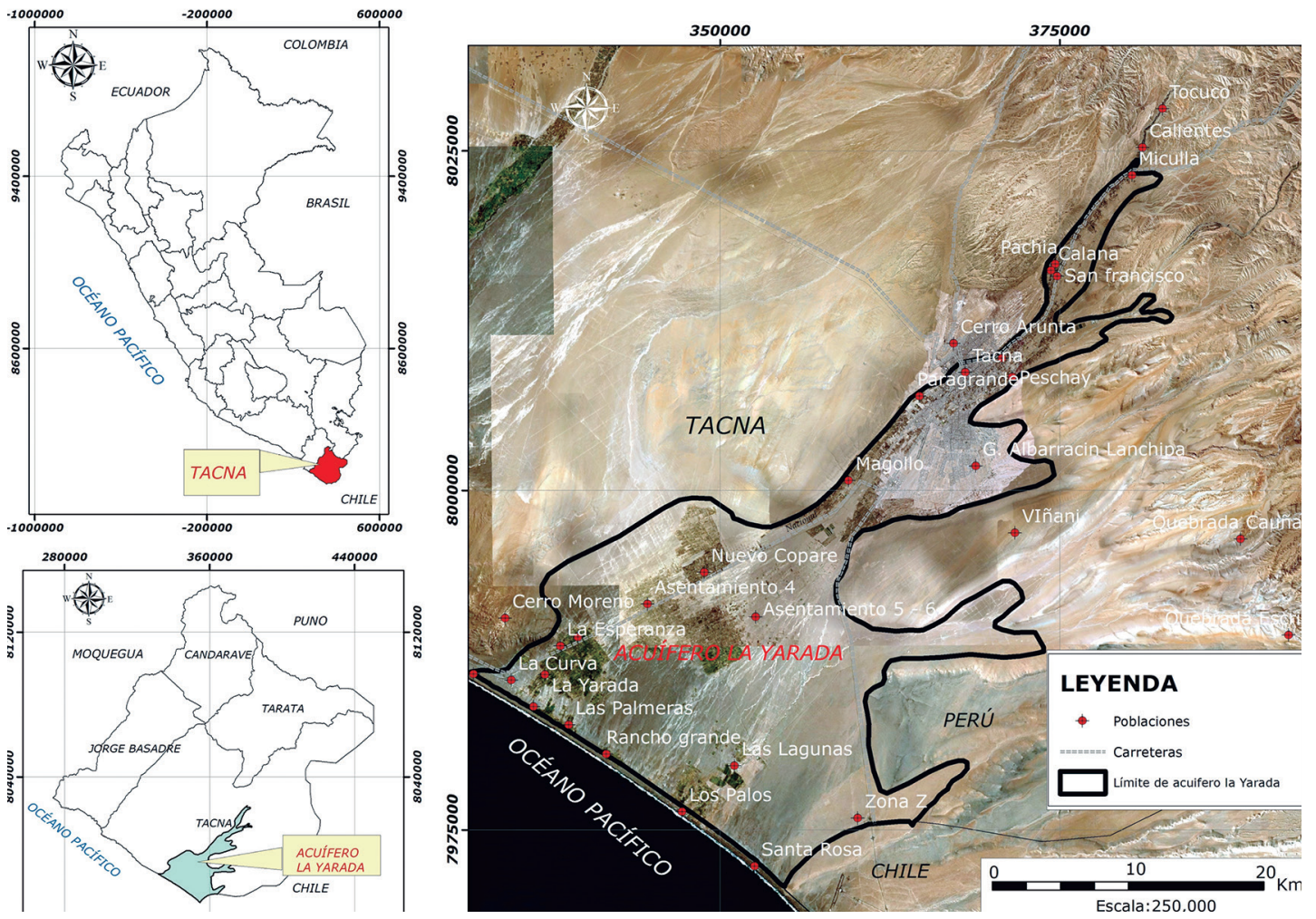

Figura 2. Mapa de ubicación del acuífero La Yarada. 


\section{En la década de 1930-1940}

Entre los años 1930 y 1940, el Ministerio de Fomento y Obras Públicas realiza el estudio para el afianzamiento de los recursos hídricos en Tacna. Se efectuaron las primeras excavaciones y se hizo el primer estudio para la utilización de aguas subterráneas, realizado por García en 1937. De esta manera podemos citar los estudios de Adams (1906), García (1933, 35 y 36) y Conklin (1939), que se ocupan de la procedencia, distribución y explotación de las aguas de escorrentía y del subsuelo de la región (Jaén et al., 1963). Entre 1940 y 1960, Petersen y Alberca (1954), Castro (1959) y Solignac (1958) efectuaron estudios en el subsuelo del valle de Tacna.

La inquietud científica por las investigaciones de las aguas subterráneas en el Perú data del año 1939, cuando Harold Conkling hizo la primera publicación del estudio sistemático de carácter general sobre la explotación de las aguas subterráneas en la costa del Perú (Conkling et al., 1939), que incluyó el acuífero costero La Yarada y donde ya se indicaba la necesidad de trazar planes para las investigaciones futuras a fin de aprovechar racionalmente este valioso recurso natural.

\section{En la década de 1950}

En 1950 se inicia el desarrollo de proyectos de irrigación privados, con las concesiones agrícolas del Ing. J. Grande y la ejecución de obras de perforación de pozos tubulares y profundos. En el año 1957 comenzaron las investigaciones de las aguas subterráneas por el U.S. Geological Survey y por el Dr. M. Solignac, experto francés en aguas subterráneas de la FAO (Food Agricultural Organization), a pedido del Ministerio de Fomento y Obras Públicas del Gobierno peruano. En la región Tacna se atendieron los requerimientos para desarrollar agricultura bajo riego usando aguas subterráneas. Las aguas superficiales siempre fueron limitadas en cantidad, por distribución espacial y temporal, además de presentar problemas de calidad por arsénico, boro y metales pesados (Pino et al., 2017). En esta década se inician las actividades agrícolas con cultivos de maíz, alfalfa y olivo, principalmente, este último en mayor proporción en vista de su bajo requerimiento de agua.

\section{En la década de 1960}

El año 1961 el Ing. C. Torres, director de proyectos del ex Ministerio de Fomento y Obras Públicas, fue encargado de crear la comisión de aguas subterráneas y en su gestión se firmó un convenio con el gobierno francés que posibilitó la venida al Perú de varios hidrogeólogos franceses como el Dr. Pierre Taltase y los ingenieros Jacques Delpy y Hubert Sommelet. También se enviaron profesionales peruanos becados a Francia para participar en programas de capacitación.

Es en 1960 cuando se inicia la ejecución de estudios hidrogeológicos en el acuífero de La Yarada. El primero de ellos fue desarrollado por el Ing. G. Pérez y culminado en diciembre de 1966, por encargo del Instituto Nacional de Investigación y Fomento Minero (INIFM). Entre sus principales conclusiones se menciona que el volumen explotado ascendía a $11.38 \mathrm{Hm}^{3} /$ año, de 63 pozos perforados solo se encontraban en actividad 34 (27 tubulares y 7 a tajo abierto) y que la reserva total de la napa era desconocida.

\section{En la década de 1970}

En 1970 se inicia el incremento de la superficie irrigada y con esto el aumento de pozos. Se explotaron aguas subterráneas para las nuevas superficies de los Asentamientos 3 y 4 y los Asentamientos 5 y 6 . En este período se perforaron los primeros piezómetros, con la finalidad de iniciar los programas de monitoreo y control del acuífero. Asimismo, se elaboró el primer modelo matemático de flujo subterráneo para el acuífero de La Yarada. Un modelo de este tipo es una versión simplificada del sistema real que simula aproximadamente los flujos de entrada-salida y las relaciones de respuesta del sistema (Thangarajan, 2007).

\section{En la década de 1980}

En 1980, la empresa Aguas Subterráneas Consultoría y Servicios S. A. (ASCOSESA) investiga el efecto de la interfase marina frente a la implementación de obras de captación en las cercanías del litoral correspondiente a la franja litoral del cono deyectivo del río Caplina. Se determina la posición y forma de avance en esta interfase frente a las condiciones de explotación. 
La Corporación Departamental de Desarrollo de Tacna (CORDETACNA) crea el Proyecto Integral La Yarada (PILY) y realiza el control de acuífero en 1985, con su respectivo inventario de recursos hídricos subterráneos. Se inventariaron 222 fuentes, de las cuales, 53 son utilizadas, 37 utilizables y 21 piezómetros de control. El resto son no utilizables. La sobreexplotación del acuífero es de alrededor de los $74 \mathrm{Hm}^{3}$ /año y se dan los primeros indicios de descensos de nivel freático. El estudio hidrogeológico realizado por INRENA en 1985 concluye que la contaminación del agua subterránea en la cercanía al litoral se debe principalmente a la intrusión marina. En la zona de La Yarada Antigua la intrusión llega hasta $\operatorname{los} 2.5 \mathrm{~km}$ y en el sector de Los Palos la contaminación presenta el doble efecto provocado por la intrusión marina y la disolución de sales concentrada en los boratos carbonatados y otros, por lo que muestran una contaminación de $3 \mathrm{~km}$ de avance hacia el continente.

Con fecha 5 de diciembre de 1989, mediante Resolución Ministerial No 0555-89-AG/DGAS, se prohíbe todo tipo de obras destinadas al alumbramiento de aguas subterráneas en las pampas de La Yarada y Hospicio, al haber determinado el Programa Nacional de Aguas Subterráneas y Tecnificación de Riego (PRONASTER), en su Estudio Integral de la Problemática de La Yarada Antigua, una sobreexplotación del acuífero, facilitando la salinización de sus aguas dulces.

\section{En la década de 1990}

En esta década, ya con un acuífero con claros indicios de sobreexplotación, que se refleja en los descensos de los niveles piezométricos, se emite la Resolución Ministerial No 621-94-AG, con fecha 5 de octubre de 1994, que autoriza al Instituto Nacional de Recursos Naturales (INRENA) para culminar el estudio hidrogeológico de las pampas de La Yarada y Hospicio, el cual se aprueba mediante Resolución Ministerial N ${ }^{\circ}$ 0696-98AG, con fecha 14 de diciembre de 1998. Allí se constata el gradual y permanente descenso del nivel freático y la intrusión marina irreversible, por lo que se recomienda mantener la veda sobre el incremento de explotación del agua subterránea. Asimismo se prohíbe ejecutar todo tipo de obra destinada a explotación.

\section{En la década del año 2000}

A raíz de los estudios y alertas de la década anterior, en el año 2004 se programa el control de acuífero en convenio entre el Proyecto Especial Tacna (PET) y la Dirección Regional de Agricultura Tacna (DRA-Tacna). En el año 2008 el INGEMMET y PET realizan el Estudio Hidrogeológico de Compatibilización del Reservorio Acuífero La Yarada. En 2003 el INRENA desarrolla el Estudio Hidrogeológico Valle del Caplina.

En el año 2009 la Autoridad Nacional del Agua (ANA) realiza el estudio Caracterización Hidrogeológica del acuífero del valle del Caplina - La Yarada. En cuanto a los asuntos de gestión del acuífero, se emite el D.S. N ${ }^{\circ}$ 065-2006AG, el cual establece la veda en el acuífero y prohíbe la ejecución de obras destinadas a la explotación de recursos hídricos subterráneos, así como el incremento de los volúmenes de explotación. Mediante R.J. No 0327-2009-ANA se ratifica la declaratoria de veda dada con el D.S. $\mathrm{N}^{\circ}$ 065-2006-AG.

\section{En la década del año 2010}

En el año 2010 el ANA desarrolla el estudio Caracterización Hidrogeoquímica del Acuífero Caplina. Asimismo, en 2011, realiza el Informe Técnico $\mathrm{N}^{\circ} 03$ sobre Calidad de agua en la Cuenca Caplina. En el año 2013 el PET elabora el diagnóstico situacional del acuífero Caplina en el ámbito del sector La Yarada.

Al ver comprometida la estabilidad del sistema, y evidenciándose el proceso de intrusión marina en el acuífero, el ANA inicia estudios orientados a la identificación de alternativas superficiales y subterráneas, tendientes a mejorar la oferta hídrica para el Caplina en el año 2013. En el año 2014, con fines de contener la intrusión marina, se propone el plan de trabajo para la implementación de una barrera hidráulica y el diagnóstico para su ubicación preliminar.

\section{Agradecimiento}

Este trabajo ha sido desarrollado en el marco del proyecto de investigación "Integración de métodos hidrodinámicos, hidroquímicos e isotópicos para precisar el funcionamiento y manejo sostenible del acuífero La Yarada, Tacna, Perú”, financiado con fondos de canon, sobrecanon y regalías mineras, de 
la Universidad Nacional Jorge Basadre Grohmann, Tacna, Perú.

\section{Discusión}

Según la normatividad y los estudios realizados para el acuífero La Yarada, durante el mencionado periodo de más de 100 años, hasta la década de los 70 la explotación del sistema estaba equilibrada entre recarga y extracciones. A partir de los 80, se concluye que la sobreexplotación del acuífero ha provocado descensos del nivel freático y que la potabilidad del agua considerada aceptable cambia a mediocre, evidenciando el deterioro en la calidad. Esto está directamente relacionado con el incremento de la actividad agrícola y la perforación y operatividad de nuevos pozos, lo cual ha redundado en la disminución progresiva de las recargas secundarias de agua de buena calidad, perjudicando a algunas zonas del acuífero. Estas se ven limitadas a recibir la recarga principal del río Caplina, donde existe actividad volcánica en las nacientes de la cuenca, lo que trae como resultado el deterioro de la calidad del agua. Pese a todo, la clasificación de agua para riego se ha mantenido invariable con el transcurso de los años (Pino \& Coarita, 2018).

En la última década, el ANA propone medidas de remediación vía recarga artificial del acuífero, con la utilización de alternativas para aumentarla, como trasvases, tratamiento de laderas, reutilización de aguas servidas y desalación. También plantea avanzar en el camino para iniciar nuevas líneas de investigación que desarrollen la técnica de recarga artificial de acuíferos. Asimismo propone estudiar y presentar ciertos criterios de educación ambiental, sensibilización y divulgación, aplicados a la recarga artificial de acuíferos. Finalmente sugiere una serie de actuaciones para acercar esta alternativa de gestión hídrica a la población en general, universidades, institutos y colegios.

También se plantea elaborar los estudios e implementar una barrera hidráulica consistente en una batería de pozos que permitan atenuar e interceptar el proceso de intrusión marina en el acuífero de La Yarada. Se enfoca en los estudios geológicos y geofísicos suficientes que permitan caracterizar plenamente el medio acuífero en la zona de implementación de esta barrera. Asimismo se centra en caracterizar químicamente el agua subterránea e identificar la zona de interfase agua dulce-agua salada. A la vez se propone establecer la ubicación y número de pozos de los cuales estaría compuesta la barrera hidráulica, además de fijar lineamientos y costos de operación y mantenimiento. Finalmente, presenta un diagnóstico para la ubicación preliminar de un piloto de barrera hidráulica negativa en el acuífero de La Yarada, con el propósito de contener la intrusión marina. Las barreras hidráulicas negativas que interceptan el flujo de agua salada mediante bombeo cerca de la costa, se han propuesto como una medida correctiva para la intrusión de agua de mar en los casos en que se deben mantener cargas piezométricas bajas (Pool \& Carrera, 2010). Las medidas correctivas deben estar diseñadas de manera óptima para mejorar la calidad del agua y minimizar los cambios en el régimen de bombeo existente (Abarca et al., 2006).

\section{Conclusión}

Tras revisar el historial de intervenciones sobre el acuífero La Yarada, a los 100 años de explotación continua en régimen equilibrado y no equilibrado, se ha determinado que la explotación no equilibrada se viene dando desde la década de los 80, en la que los volúmenes de extracción superan ampliamente a los de recarga. Por parte de los gestores del agua en el acuífero, se han tomado medidas acertadas, tales como las vedas, y no acertadas, como la emisión de decretos de regularización de pozos no autorizados, que incentivaron las perforaciones en forma indiscriminada con el fin de legalizarlas. Se identificaron los problemas técnicos respecto a la sobreexplotación del acuífero y se han dado lineamientos actuales y futuros para fijar políticas respecto a su explotación, así como las condiciones de esta en términos de cantidad y calidad. A futuro, se plantearon medidas correctoras como el empleo de barreras hidráulicas negativas, positivas y mixtas, plenamente justificadas con la finalidad de atenuar los efectos de la intrusión marina, que viene contaminando el agua subterránea del acuífero. 


\section{Literatura Citada}

Abarca, E.; Vázquez-Suñé, E.; Carrera, J.; Capino, B.; Gámez, D.; Batlle, F.

2006. Optimal design of measures to correct seawater intrusion: measures to correct seawater intrusion. Water Resources Research, 42(9). doi:10.1029/2005WR004524.

Arújo, L.M.; França, A.B.; Potter, P.E.

1999. Hydrogeology of the Mercosul aquifer system in the Paraná and Chaco-Paraná Basins, South America, and comparison with the Navajo-Nugget aquifer system, USA. Hydrogeology Journal, 7(3): 317-336.

Babaye, M.S.A.; Orban, P.; Ousmane, B.; Favreau, G.; Brouyère, S.; \& Dassargues, A.

2018. Characterization of recharge mechanisms in a Precambrian basement aquifer in semi-arid south-west Niger. Hydrogeology Journal, 27(2): 475-491.

Conkling, H.; Góngora Trejos, E.

1939. Explotación de aguas subterráneas en la costa del Perú. Sociedad Nacional Agraria. Lima. Perú. 263 p.

Döll, P.; Hoffmann-Dobrev, H.; Portmann, F.T.; Siebert, S.; Eicker, A.; Rodell, M.; Scanlon, B.R.

2012. Impact of water withdrawals from groundwater and surface water on continental water storage variations. Journal of Geodynamics, 59-60: 143-156.

Jaén, H.; Ortiz, G.; Wilson, J.

1963. Geología. Cuadrángulo de La Yarada y Tacna. Boletín $\mathrm{N}^{\circ}$ 6. Comisión de la Carta Geológica Nacional. Lima, Perú. 75 p.

Margat, J.; Gun, J. van der.

2013. Groundwater around the world: a geographic synopsis. CRC. Boca Raton, Fla, USA. 376 p.

Montaño Xavier, J.; Tujchneider, O.C.; Auge, M.; Fili, M.; Paris, M.; D’Elía, M.; Pérez, M.; Nagy, M.; Collazo, P.; Decoud, P. 1998. Sistema acuífero guaraní: Acuíferos regionales en América Latina; capítulo argentino - uruguayo. Univ. Nal. del Litoral. Santa Fe, Argentina. 216 p.

Nicholson, S.

2001. Climatic and environmental change in Africa during the last two centuries. Climate Research, 17: 123-144.

Pino, E.; Tacora, P.; Steenken, A.; Alfaro, L.; Valle, A.; Chávarri, E.; Mejía, J.
2017. Efecto de las características ambientales y geológicas sobre la calidad del agua en la cuenca del río Caplina, Tacna, Perú. Tecnología y Ciencias del Agua, 08(06): 77-99.

Pino, V.E.; Chávarri, V.E.; Ramos, F.L.

2018. Crisis de gobernanza y gobernabilidad y sus implicancias en el uso inadecuado del agua subterránea, caso acuífero costero de La Yarada, Tacna, Perú. Idesia (Arica), 36(3): 75-85.

Pino V.E.; Coarita A.F.

2018. Caracterización hidrogeológica para determinar el deterioro de la calidad del agua en el acuífero La Yarada Media. Revista de Investigaciones Altoandinas -Journal of High Andean Research, 20(4): 477-490.

Pool, M.; Carrera, J.

2010. Dynamics of negative hydraulic barriers to prevent seawater intrusion. Hydrogeology Journal, 18(1): 95-105.

Post, V.E.A.

2005. Fresh and saline groundwater interaction in coastal aquifers: Is our technology ready for the problems ahead? Hydrogeology Journal, 13(1): 120-123.

Schneider, S.H.; Root, T.L.; Mastrandrea, M.D.

2011. Encyclopedia of Climate and Weather. 2. ${ }^{\mathrm{a}}$ ed. Oxford University Press. Oxford, UK. 531 p.

Shiklomanov, A.; Rodda, J.

2004. World Water Resources at the Beginning of the TwentyFirst Century. 13. Cambridge University Press. UK. 253 p.

Taylor, R.G.; Todd, M.C.; Kongola, L.; Maurice, L.; Nahozya, E.; Sanga, H.; MacDonald, A.M.

2012. Evidence of the dependence of groundwater resources on extreme rainfall in East Africa. Nature Climate Change, 3: 374.

Thangarajan, M.

2007. Groundwater Models and Their Role in Assessment and Management of Groundwater Resources and Pollution. En: Thangarajan, M. Ed. Groundwater: Resource Evaluation, Augmentation, Contamination, Restoration, Modeling and Management. SpringerNetherlands. New Delhi, India. pp. 189-236.

Zagal, R.; Galdames, L.; Díaz Araya, A.

2015. Junta de Adelanto de Arica (1958-1976). Experiencia, documentos e historia regional. Ediciones Universidad de Tarapacá. Arica, Chile. 171 p. 
